\title{
Cones in the Euclidean space with vanishing scalar curvature
}

\author{
JOÃO LUCAS M. BARBOSA ${ }^{1 *}$ and MANFREDO DO CARMO ${ }^{2 *}$ \\ ${ }^{1}$ Universidade Federal do Ceará (UFC), Departamento de Matemática \\ Campus do Pici, 60455-760 Fortaleza, Ce, Brasil \\ ${ }^{2}$ Instituto Nacional de Matemática Pura e Aplicada (IMPA) \\ Estrada Dona Castorina 110, 22460-320 Rio de Janeiro, RJ, Brasil
}

Manuscript received on April 22, 2004; accepted for publication on May 12, 2004.

\begin{abstract}
Given a hypersurface $M$ on a unit sphere of the Euclidean space, we define the cone based on $M$ as the set of half-lines issuing from the origin and passing through $M$. By assuming that the scalar curvature of the cone vanishes, we obtain conditions under which bounded domains of such cone are stable or unstable.
\end{abstract}

Key words: stability, $r$-curvature, cone, scalar curvature.

\section{INTRODUCTION}

A natural generalization of minimal hypersurfaces in Euclidean spaces was introduced in (Reilly 1973). Reilly considered the elementary symmetric functions $S_{r}, r=0,1, \ldots, n$, of the principal curvatures $k_{1}, \ldots, k_{n}$ of an orientable hypersurface $x: M^{n} \rightarrow R^{n+1}$ given by

$$
S_{0}=1, \quad S_{r}=\sum_{i_{1}<\cdots<i_{r}} k_{i_{1}} \ldots k_{i_{r}}
$$

Here, $k_{i_{1}}, \ldots, k_{i_{n}}$ are the eigenvalues of $A=-d g$, where $g: M^{n} \rightarrow S^{n}(1)$ is the Gauss map of the hypersurface. Reilly showed that orientable hypersurfaces with $S_{r+1}=0$ are critical points of the functional

$$
\mathcal{A}_{r}=\int_{M} S_{r} d M
$$

for variations of $M$ with compact support. Thus, such hypersurfaces generalize the fact that minimal hypersurfaces are critical points of the area functional $A_{0}=\int_{M} S_{0} d M$ for compactly supported variations.

\footnotetext{
*Member Academia Brasileira de Ciências

Correspondence to: João Lucas M. Barbosa

E-mail: jlucas@secrel.com.br
} 
A breakthrough in the study of these hypersurfaces occurred in the last five years of last century: in (Hounie and Leite 1995) and (Hounie and Leite 1999) conditions for the linearization of the partial differential equation $S_{r+1}=0$ to be an elliptic equation were found. This linearization involves a second order differential operator $L_{r}$ (see the definition of $L_{r}$ in Section 2) and the Hounie-Leite conditions read as follows:

$$
L_{r} \text { is elliptic } \Longleftrightarrow \operatorname{rank}(A)>r+1 \quad \Longleftrightarrow \quad S_{r+2} \neq 0 \text { everywhere. }
$$

In this paper, we will be interested in the case $S_{2}=0$. For this situation, since $\operatorname{rank}(A)$ cannot be two, the ellipticity condition is equivalent to rank $(A) \geq 3$.

In (Alencar et al. 2003) a general notion of stability was introduced for bounded domains of hypersurfaces of Euclidean spaces with $S_{r+1}=0$. In the case we are interested, namely $S_{2}=0$, it can be shown that if we assume that $L_{1}$ is elliptic, an orientation can be chosen so that a bounded domain $D \subset M$ is stable if

$$
\left.\frac{d^{2} A_{1}}{d t^{2}}\right|_{t=0}>0 \quad \text { for all variations with support in (the open set) } D \text {. }
$$

In what follows, we denote by $B_{r}(0)$ the ball of radius $r$ centered at the origin 0 of $R^{n+1}$. Let $M^{n-1}$ be a smooth hypersurface of the sphere $S^{n}(1)$. A cone $\mathcal{C}(M)$ in $R^{n+1}$ is the union of half-lines starting at 0 and passing through the points of $M$. It is clear that $\mathcal{C}(M) \cap S^{n}(1)=M$. It is easy to show that $\mathcal{C}(M)-\{0\}$ is a smooth $n$-dimensional hypersurface of $R^{n+1}$. The manifold $\mathcal{C}(M)$ is referred to as the cone based on $M^{n-1}$. The part of the cone contained in the closure of the ring $B_{1}(0) \backslash B_{\varepsilon}(0), 0<\varepsilon<1$, is called a truncated cone and is denoted by $\mathcal{C}(M)_{\varepsilon}$.

In this note we present the following two theorems which provide a nice description of the stability of truncated cones in $R^{n+1}$ based on compact, orientable hypersurfaces of $S^{n}(1)$, with $S_{2}=0$ and $S_{3} \neq 0$ everywhere.

THEOREM 1. Let $M^{n-1}, n \geq 4$, be an orientable, compact, hypersurface of $S^{n}(1)$ with $S_{2}=0$ and $S_{3} \neq 0$ everywhere. Then, if $n \leq 7$, there exists an $\varepsilon>0$ so that the truncated cone $\mathcal{C}(M)_{\varepsilon}$ is not stable.

THEOREM 2. For $n \geq 8$, there exist compact, orientable hypersurfaces $M^{n-1}$ of the sphere $S^{n}(1)$, with $S_{2}=0$ and $S_{3} \neq 0$ everywhere, so that, for all $\varepsilon>0, \mathcal{C}(M)_{\varepsilon}$ is stable.

Although Theorems 1 and 2 are interesting on their own right, a further motivation to prove these theorems is that, for the minimal case, they provide the geometric basis to prove the generalized Bernstein theorem, namely, that a complete minimal graph $y=f\left(x_{1}, \ldots, x_{n-1}\right)$ in $R^{n}, n \leq 8$, is a linear function (See (Simons 1968), Theorems 6.1.1, 6.1.2, 6.2.1, 6.2.2).

For elliptic graphs in $R^{n}$ with vanishing scalar curvature the question appears in a natural way. Of course, since we want to consider graphs with $S_{2}=0$ and $S_{3}$ never zero, we must start with $n \geq 4$, and the solution cannot be a hyperplane. Thus the question is whether there exists an elliptic graph in $R^{n}, n \geq 4$, with vanishing scalar curvature. 


\section{PRELIMINARIES}

For notational reasons, it will be convenient to denote the hypersurface of the Introduction by $x: \bar{M} \rightarrow R^{n+1}$. We first need to consider the Newton Transformations $P_{r}$, that are inductively given by

$$
\begin{aligned}
& P_{0}=I \\
& P_{r}=S_{r} I-A P_{r-1},
\end{aligned}
$$

and then define the differential operator $L_{r}$ by

$$
L_{r} f=\operatorname{trace}\left\{P_{r} \operatorname{Hess} f\right\} .
$$

It turns out that $L_{r}$ is self-adjoint and that $L_{r} f=\operatorname{div}\left(P_{r} \operatorname{grad} f\right)$.

The second variation formula for the variational problem of the functional $\mathcal{A}_{1}$ is, up to a positive constant, given by

$$
I(f)=-\int_{\bar{M}} f\left(L_{1} f-3 S_{3} f\right) d \bar{M},
$$

for test functions $f$ of compact support in $\bar{M}$.

Consider now a compact orientable $(n-1)$-dimensional manifold $M$ immersed as a hypersurface of the unit sphere $S^{n}(1)$ of the Euclidean space $R^{n+1}$. The cone $\mathcal{C}(M)$ based on $M$ is described by

$$
\begin{aligned}
M \times(0, \infty) & \rightarrow R^{n+1} \\
(m, t) & \rightarrow t m
\end{aligned}
$$

Of course, the geometry of $\mathcal{C}(M)$ is closely related to the one of $M$ and it is simple to compute the second fundamental form $\bar{A}$ of $\mathcal{C}(M)$ in terms of the second fundamental form $A$ of $M$. In fact, one finds

$$
\bar{A}=\frac{1}{t} A .
$$

From this relation on the second fundamental forms it follows that

Proposition 1. If $\bar{S}_{r}$ represents the elementary symmetric function of order $r$ of $\mathcal{C}(M)$ and $\bar{P}_{r}$ its Newton transformations, then:

a) $\bar{S}_{r}=\left(1 / t^{r}\right) S_{r}$,

b) $\bar{S}_{r}=0 \quad$ if and only if $\quad S_{r}=0$,

c) $|\bar{A}|=(1 / t)|A|$, 
d) $\bar{P}_{r}=\left(1 / t^{r}\right)\left[\begin{array}{rrc}S_{r} & 1 & 0 \\ --- & -+- & ---- \\ & 1 & \\ 0 & 1 & P_{r}\end{array}\right]$.

Proof. The proof is direct except for the last item. But this can be done using finite induction and the definition of $\bar{P}_{r}$.

Let $F: \mathcal{C}(M) \rightarrow R$ be a $C^{2}$ function. For each $t>0$, define $\tilde{F}_{t}: M \rightarrow R$ by $\tilde{F}_{t}(m)=F(m, t)$. Proposition 2. With the above notation we have:

$$
\bar{L}_{r} F=\frac{1}{t^{r}} S_{r} \frac{\partial^{2} F}{\partial t^{2}}+\frac{n-r-1}{t^{r+1}} S_{r} \frac{\partial F}{\partial t}+\frac{1}{t^{r+1}} L_{r}\left(\tilde{F}_{t}\right)
$$

PROOF. The proof of this Lemma follows the same lines used to find the expression of the Laplacian in polar coordinates and using the previous proposition.

\section{SKETCH OF PROOF OF THEOREM 1}

First of all let us observe that since $S_{2} \equiv 0$ then $\left(S_{1}\right)^{2}=|A|^{2} \geq 0$. Hence, at a point where $S_{1}=0$ we would have that all the entries of the matrix $A$ are zero and so $S_{3}=0$ what is forbidden by our hypothesis. Therefore, we will have $\left(S_{1}\right)^{2}>0$ everywhere.

According to Proposition 1, our hypotheses then imply that, for the cone $\mathcal{C}(M)$, we have $\bar{S}_{2} \equiv 0$ and $\bar{S}_{1}$ and $\bar{S}_{3}$ never zero.

It was proved in (Hounie and Leite 1999) that, for a hypersurface of $R^{n+1}$ with $\bar{S}_{r} \equiv 0$, $2 \leq r<n$, the operator $\bar{L}_{r-1}$ is elliptic if and only if $\bar{S}_{r+1}$ is never zero. Then we conclude that $L_{1}$ and $\bar{L}_{1}$ are elliptic.

To prove the theorem, we are going to show the existence of a truncated cone $\mathcal{C}(M)_{\epsilon}$ for which the second variation formula attains negative values. Hence, from now on we are going to work on a truncated cone, with test functions $f$ that have a support contained in the interior of the truncated cone. As we did before, for each test function $f: \mathcal{C}(M)_{\epsilon} \rightarrow R$ and each fixed $t$ we define $\tilde{f}_{t}: M \rightarrow R$ by $\tilde{f}_{t}(m)=f(m, t)$. From Proposition 2 we have that

$$
\bar{L}_{1} f=\frac{1}{t} S_{1} \frac{\partial^{2} f}{\partial t^{2}}+\frac{n-2}{t^{2}} S_{1} \frac{\partial f}{\partial t}+\frac{1}{t^{3}} L_{1}\left(\tilde{f}_{t}\right) .
$$

The volume element of $\mathcal{C}(M)$ is easily seen to be

$$
d \bar{M}=t^{n-1} d t \wedge d M
$$


Hence, using (3), (5) and the expression of the volume, the second variation formula on $f$ becomes

$$
\begin{aligned}
I(f)= & -\int_{M \times[\epsilon, 1]}\left(\tilde{f}_{t} L_{1}\left(\tilde{f}_{t}\right)-3 S_{3}\left(\tilde{f}_{t}\right)^{2}\right) t^{n-4} d t \wedge d M- \\
& -\int_{M \times[\epsilon, 1]}\left(t^{2} f \frac{\partial^{2} f}{\partial t^{2}}+(n-2) t f \frac{\partial f}{\partial t}\right) t^{n-4} S_{1} d t \wedge d M .
\end{aligned}
$$

Since $S_{1}>0$, then $t^{n-4} S_{1} d t \wedge d M$ is a volume element in $\mathcal{C}(M)$, in particular in $\mathcal{C}(M)_{\epsilon}$. We will represent it by $d S$. In fact, $d S$ is a product of two measures. The first one on the real line: $d \xi=t^{n-4} d t$; the second, on $M$, given by $d \mu=S_{1} d M$. So, $d S=d \xi \wedge d \mu$. We can then rewrite the second variation formula on $f$ as:

$$
\begin{aligned}
I(f)= & -\int_{M \times[\epsilon, 1]} \frac{1}{S_{1}}\left(\tilde{f}_{t} L_{1}\left(\tilde{f}_{t}\right)-3 S_{3}\left(\tilde{f}_{t}\right)^{2}\right) d \xi \wedge d \mu \\
& -\int_{M \times[\epsilon, 1]}\left(t^{2} f \frac{\partial^{2} f}{\partial t^{2}}+(n-2) t f \frac{\partial f}{\partial t}\right) d \xi \wedge d \mu .
\end{aligned}
$$

Define, now, the following two operators:

$$
\begin{array}{lll}
\mathcal{L}_{1}: C^{\infty}(M) \rightarrow C^{\infty}(M) & \text { by: } & \mathcal{L}_{1} f=-\left(1 / S_{1}\right) L_{1} f+3\left(S_{3} / S_{1}\right) f . \\
\mathcal{L}_{2}: C^{\infty}[\epsilon, 1] \rightarrow C^{\infty}[\epsilon, 1] & \text { by: } & \mathcal{L}_{2} g=-t^{2} g^{\prime \prime}-(n-2) t g^{\prime} .
\end{array}
$$

Observe that we are considering the space $C^{\infty}(M)$ with the inner product:

$$
\ll f_{1}, f_{2} \gg=\int_{M} f_{1} f_{2} d \mu
$$

and $C^{\infty}[\epsilon, 1]$ with the inner product:

$$
\left\langle g_{1}, g_{2}\right\rangle=\int_{\epsilon}^{1} g_{1} g_{2} d \xi
$$

Since $L_{1}$ is elliptic and $M$ is compact then $L_{1}$, and so $\mathcal{L}_{1}$, is strongly elliptic. The same is true for the operator $\mathcal{L}_{2}$. Let $\lambda_{1} \leq \lambda_{2} \leq \cdots \nearrow \infty$ be the eigenvalues of $\mathcal{L}_{1}$ and $\delta_{1}<\delta_{2}<\cdots \nearrow \infty$ be the eigenvalues of $\mathcal{L}_{2}$. Using orthonormal bases of eigenfunctions for theses operators one deduces the following Lemma:

LEMMa 1. For any test function $f$ we have

$$
I(f) \geq\left(\lambda_{1}+\delta_{1}\right) \int_{M \times[\epsilon, 1]} f^{2} d \xi \wedge d \mu .
$$

There exists a test function $f$ such that $I(f)<0$ if and only if $\lambda_{1}+\delta_{1}<0$.

The operator $\mathcal{L}_{2}$ is well known. In fact it has been used in (Simmons 1968) to prove his celebrated theorem. The following lemma contains all the information we need about this operator: 
Lemma 2. The operator $\mathcal{L}_{2}$ has eigenvalues

$$
\delta_{k}=\left(\frac{n-3}{2}\right)^{2}+\left(\frac{k \pi}{\log \epsilon}\right)^{2}
$$

where $1 \leq k<\infty$.

We will also need the following lemma whose proof uses Lemmas (3.7) and (4.1) in (Alencar et al. 1993).

LEMma 3. Let $M^{n-1}$ be a compact, orientable, immersed hypersurface of $S^{n}(1)$ with $S_{2} \equiv 0$ e $S_{3}$ never zero. Suppose $n \geq 4$. The first eigenvalue of the operator $\mathcal{L}_{1}$ in $M$ satisfy: $\lambda_{1} \leq-(n-2)$.

Finally, we observe that the lemma below completes the proof of Theorem 1.

Lemma 4. Let $M^{n-1}$ be a compact, orientable, immersed hypersurface of $S^{n}(1)$ with $S_{2} \equiv 0, S_{3}$ never zero and $n \geq 4$. If $n \leq 7$ then there exists $\epsilon>0$ such that the truncated cone $\mathcal{C} M_{\epsilon}$ is not stable.

Proof of The Lemma: From Lemmas 2 and 3 we have

$$
\lambda_{1}+\delta_{1} \leq-(n-2)+\left(\frac{n-3}{2}\right)^{2}+\left(\frac{\pi}{\log \epsilon}\right)^{2} .
$$

It is trivial to verify that the sum of the first two terms of the right hand side of this inequality is a quadratic polynomial, with positive second order term, whose roots are approximately 2.2 and 7.8. Hence it is strictly negative for values of $n \in\{4,5,6,7\}$, in fact, it is less than or equal to -1 . Hence,

$$
\lambda_{1}+\delta_{1} \leq-1+\left(\frac{\pi}{\log \epsilon}\right)^{2} .
$$

Choosing $\epsilon$ sufficiently small we can guarantee that the right hand side is negative. Now, by Lemma 1 , we see that $\mathcal{C} M_{\epsilon}$ is not stable. This proves Lemma 4 and completes the proof of the Theorem 1.

\section{EXISTENCE OF STABLE CONES}

In this section we sketch the proof o Theorem 2.

The following example has been considered by various people in different contexts (see e.g. (Chern 1968) and (Alencar et al. 2002). Consider $R^{p+2}=R^{r+1} \oplus R^{s+1}, r+s=p$. Write down the vectors of $R^{p+2}$ as $\xi_{1}+\xi_{2}, \xi_{1} \in R^{r+1}, \xi_{2} \in R^{s+1}$. When $\xi_{1}$ describes $S^{r}(1) \subset R^{r+1}$ and $\xi_{2}$ describes $S^{s}(1) \subset R^{s+1}$, by taking positive numbers $a_{1}$ and $a_{2}$ with $a_{1}^{2}+a_{2}^{2}=1$, we have that

$$
x=a_{1} \xi_{1}+a_{2} \xi_{2}
$$

describes a submanifold $M$ of dimension $p=r+s$ of the sphere $S^{p+1}(1) \subset R^{p+2}$. The manifold $M$ is diffeomorphic to $S^{r}(1) \times S^{s}(1)$ and so is compact and orientable. It can be shown that $a_{1}$ and $a_{2}$ can be chosen so that $S_{2}=0$ and $S_{3} \neq 0$. We will show that, in this case, the truncated cone $\mathcal{C}(M)_{\varepsilon}$ is stable as a hypersurface of $R^{r+s+1}$ when $r+s+1 \geq 8$. 
It can be shown that the $L_{1}$ operator on $M$ is given by

$$
L_{1} f=\left[(r-1) \frac{a_{2}}{a_{1}}-s \frac{a_{1}}{a_{2}}\right] \Delta^{r} f+\left[r \frac{a_{2}}{a_{1}}-(s-1) \frac{a_{1}}{a_{2}}\right] \Delta^{s} f,
$$

where $\Delta^{r}$ and $\Delta^{s}$ denote the Laplacian operators in the Euclidean spheres $S^{r}\left(a_{1}\right)$ and $S^{s}\left(a_{2}\right)$, respectively. Since the first nonzero eigenvalue of the Laplace operator on a sphere $S^{k}(b)$ is known to be $k / b^{2}$, the first nonzero eigenvalue of $L_{1}$ will be

$$
\tilde{\lambda}_{1}=\min \left\{\left[(r-1) \frac{a_{2}}{a_{1}}-s \frac{a_{1}}{a_{2}}\right] \frac{r}{a_{1}^{2}},\left[r \frac{a_{2}}{a_{1}}-(s-1) \frac{a_{1}}{a_{2}}\right] \frac{s}{a_{2}^{2}}\right\} .
$$

It will then follow that the first eigenvalue of the operator

$$
\mathcal{L}_{1}=\frac{1}{S_{1}} L_{1}+3 \frac{S_{3}}{S_{1}}
$$

will be given by

$$
\lambda_{1}=3 \frac{S_{3}}{S_{1}}=-(p-1),
$$

where the last equality comes from a long but straightforward computation.

Therefore, using Lemma 2, the above value for $\lambda_{1}$, and the fact that, in our case, $n=p+1$, we obtain

$$
\lambda_{1}+\delta_{1}=-(n-2)+\left(\frac{n-3}{2}\right)^{2}+\left(\frac{\pi}{\log \varepsilon}\right)^{2} .
$$

For $n \geq 8$, the sum of the first two terms becomes $>1 / 4$. Thus, for any choice of $\varepsilon, \lambda_{1}+\delta_{1}>0$. Together with Lemma 1, this completes the proof of Theorem 2.

\section{ACKNOWLEDGMENTS}

Both authors were partially supported by PRONEX and CNPq.

\section{RESUMO}

Dada uma hipersuperfície $M$ de uma esfera unitária do espaço euclidiano, definimos o cone sobre $M$ como o conjunto das semi-retas que saem da origem e passam por $M$. Admitindo que a curvatura escalar de um dado cone é nula, estabelecemos condições para que os seus domínios limitados sejam estáveis ou instáveis.

Palavras-chave: estabilidade, $r$-curvatura, cone, curvatura escalar.

\section{REFERENCES}

Alencar H, do Carmo MP and Colares AG. 1993. Stable hypersurfaces with constant scalar curvature. Math Z 213: 117-131.

Alencar H, do Carmo MP and Santos W. 2002. A gap theorem for hypersurfaces of the sphere with constant scalar curvature one. Comm Math Helv 77: 549-562. 
Alencar H, do Carmo MP and Elbert MF. 2003. Stability of hypersurfaces with vanishing $r$-mean curvatures in euclidean spaces. J Reine Angew Math 554: 201-216.

CHERN SS. 1968. Minimal submanifolds in a Riemannian manifold. Lecture Notes, Lawrence, Kan., Univ. of Kansas.

Hounie J AND LeIte ML. 1995. The maximum principle for hypersurfaces with vanishing curvature functions. J Diff Geom 41: 247-258.

Hounie J AND LeITe ML. 1999. Two-ended hypersurfaces with zero scalar curvature. Indiana Univ Math J 48: 867-882.

REILLY R. 1973. Variational properties of functions of the mean curvatures for hypersurfaces in space forms. J Diff Geom 8: 465-477.

Simmons J. 1968. Minimal varieties in Riemannian manifolds. Ann Math 88: 62-105. 